\title{
Magnetic Properties of Ni Thin Films Deposited on to Polystyrene Nanospheres
}

\author{
Yogesh Kumar ${ }^{1, *}$, J. Tripathi², A. Sharma ${ }^{1, \dagger}$ \\ ${ }^{1}$ Department of Physics, Manipal University Jaipur, India \\ ${ }^{2}$ Department of Physics, ISLE, IPS Academy, Indore, India
}

(Received 15 January 2021; revised manuscript received 12 February 2021; published online 25 February 2021)

\begin{abstract}
Structural and magnetic properties of Ni magnetic nanocaps synthesized using electron beam evaporation technique under ultra high vacuum conditions are presented for two Ni layer thicknesses, namely $10 \mathrm{~nm}$ and $20 \mathrm{~nm}$. To prepare them, Si (100) substrate was first drop coated with soft polystyrene (PS) nanopheres with the average diameter of $800 \mathrm{~nm}$ followed by the evaporation of Ni onto dried substrate. For the comparative analysis, the films were simultaneously deposited on plane Si substrate. The films were characterized for their crystalline properties using X-ray diffraction measurement in grazing incidence geometry, while other structural properties such as roughness and thickness of these films were extracted from the fitting of X-ray reflectivity data. The parameters were then correlated with the magnetism observed in these films using magneto-optical Kerr effect (MOKE) technique. The influence of thickness in these properties is discussed here.
\end{abstract}

Keywords: GIXRD, MOKE, XRR, Polystyrene, Magnetic nanocaps.

DOI: 10.21272/jnep.13(1).01028

PACS numbers: 61.05.C, 75.70.i, 75.75. + a

\section{INTRODUCTION}

Magnetic nanostructures are known to exhibit new and interesting magnetic properties such as superparamagnetism, extraordinary exchange bias, large magnetic anisotropy and enhancement in coercivity etc., which make them a suitable candidate for magnetic devices comparison to their bulk materials counterpart [1,2]. The practical applications of these magnetic nanostructures, especially those of isolated magnetic nanocaps, are interesting because such nanocaps fall into the category of oriented magnetic nanostructures possessing nanoscale size and curvature dependent magnetic properties. These properties can be tailored to obtain optimized storage capacity and in some applications, optimized device speed and performance [3]. The magnetic films on flat substrates are generally used to fabricate many devices, however, if prepared on the curved surfaces, they can provide additional advantages arising from the curvature induced lateral film-thickness variation, which enables the deposition of ultra-thin to thin film on a single curvature where their combined magnetic effect is observed depending upon the type of deposited film material $[4,5]$. In order to prepare efficient, high performance magnetic devices fabricated using such nanostructures, several lithographic methods have been utilized in the past. These fabrication methods provide good control over various geometric factors such as growth morphology, crystallite size orientation, magnetic domain orientation and magnetic anisotropy etc. In particular, nanosphere lithography technique (also called as natural lithography) has attracted more attention in recent years in comparison with other conventional lithography techniques. This is due to its cost effectiveness, easy fabrication procedure, fast preparation and uniform coating of nanospheres over a wide substrate surface area [6]. This technique is specifically useful for the synthesis of "magnetic nanocaps", which consist of isolated nanomagnets having negligible or limited dipole and exchange interaction among nearest neighbors [7]. The magnetic nanocaps made from Co and Co based magnetic nanostructures have attracted a lot of attention for their possible applications in next generation magnetic storage devices [7, 9]. Yet, other magnetic material including $\mathrm{Ni}$ may also be useful for such purpose and a need to explore them is felt. Although a few reports on $\mathrm{Ni}$ based structures for these applications are present in literature, the topic has not been much explored. M. Hemmous et al. used vibrating sample magnetometer set-up to investigate the thickness dependent (4 to $163 \mathrm{~nm}$ magnetic properties of evaporated $\mathrm{Ni}$ thin films deposited on different substrates. It was found that variation in substrate material induces changes in coercive field as well as in the origins of these values. The squareness also depends on substrate and thickness [8].

L. Kerkache et al. also studied the thickness and substrate materials dependent magnetic properties of $\mathrm{Ni}$ thin films deposited onto various substrates (glass, $\mathrm{Si}$ single crystal, mica and $\mathrm{Cu}$ ), in which thicknessdependent stress induced anisotropy was observed [10]. In the present work, we have prepared curved surface on plain standard substrate and study the magnetic and structural properties of Ni nanocaps evaporated on this curved surface. A comparison of the properties of nanocaps of varying thicknesses on a curved surface are compared to those of the same thickness films deposited on reference flat substrate.

\section{EXPERIMENTAL}

\subsection{Thin Film Deposition}

The Si (100) substrate was thoroughly cleaned by using acetone followed by cleaning with methanol. The

\footnotetext{
* yogeshamity91@gmail.com

† anupamcsr@gmail.com

The results were presented at the International Conference on Multifunctional Nanomaterials (ICMN2020)
} 
curved substrates containing nanospheres were prepared by nanosphere lithography technique. For this purpose, a self-assembled polystyrene (PS) nanosphere layer with a diameter of $\sim 800 \mathrm{~nm}$ was first deposited on cleaned $\mathrm{Si}$. This curved surface was then made available for depositing $\mathrm{Ni}$ with two different thicknesses of $10 \mathrm{~nm}$ and $20 \mathrm{~nm}$ with the help of electron beam evaporation method under ultra-high vacuum condition. The base pressure in the electron beam evaporation deposition chamber was $6.5 \cdot 10^{-7}$ Torr, which during deposition increased to $7.7 \cdot 10^{-6}$ Torr. The deposition rate of $\mathrm{Ni}$ was $0.6 \mathrm{~nm} / \mathrm{min}$. In addition, at the same time, the reference films of $\mathrm{Ni}$ were also simultaneously deposited directly on Si substrate. The schematic of Ni film grown on plane Si substrate and on PS arrays is depicted in Fig. 1.

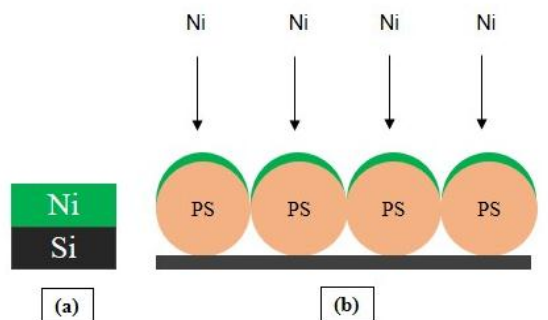

Fig. 1 - Schematic of growth of the Ni film on (a) plane $\mathrm{Si}$ substrate and (b) polystyrene nanospheres coated on Si substrate

\subsection{Characterization Techniques}

The magneto-optical Kerr effect (MOKE) technique was used to measure the magnetic properties of $\mathrm{Ni}$ thin film on both plain and curved surfaces. He-Ne laser of wavelength $632.8 \mathrm{~nm}$ was used for these measurements. For measurement of structural properties, the X-ray reflectivity (XRR)and the Grazing incident X-ray diffraction (GIXRD) techniques were used with the same CuKa $(\lambda=0.154 \mathrm{~nm})$ source on Bruker D8discover machine (operated at $40 \mathrm{kV}, 30 \mathrm{~mA}$ ). FESEM image of the synthesized PS nanosphere array on $\mathrm{Si}$ substrate was recorded using JEOL 7610FPlus design instrument. Scanning electron microscopy (SEM) was used to study morphology of synthesized self-assembly of the nanospheres.

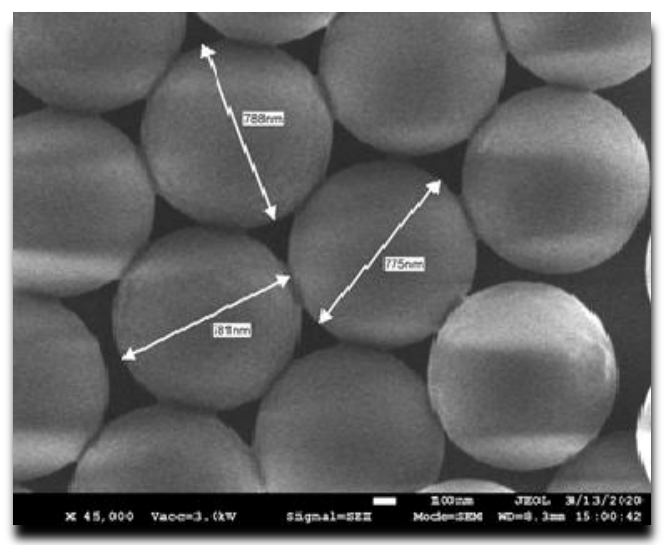

Fig. 2-FESEM 2D image of polystyrene nanosphere synthesized on Si substrate showing the average diameter of $\sim 800 \mathrm{~nm}$
FESEM image revealed uniform and monodisperse distribution of the PS nanospheres have with an average diameter of $800 \mathrm{~nm}$ on $\mathrm{Si}(100)$ substrate (Fig. 2). The arrangement of these nanospheres is hexagonal on the surface and their boundaries touch each other. However, their upper surfaces are separated from those of surrounding nanospheres.

\section{RESULTS AND DISCUSSION}

\subsection{Grazing Incidence X-ray Diffraction (GIXRD) Technique}

Fig. 3 shows the GIXRD patterns recorded in the $2 \theta$ range from 35 to $80^{\circ}$ on both types of Ni films: (a) deposited on polystyrene and (b) deposited on Si substrates. The Bragg reflection arising from $\mathrm{Ni}(111)$ and $\mathrm{Ni}(200)$ fcc fundamental peaks at $2 \theta=44.2^{\circ}$ and $53.5^{\circ}$, respectively, are clearly visible in $\mathrm{Ni} / \mathrm{Si}$ sample, showing polycrystalline nature of the grown films [11]. On the other hand, in Ni/PS samples, the XRD peaks of $\mathrm{Ni}$ (111) plane are not clearly visible due to low intensity, indicating a change in relative crystallinity when the underlying surface is changed. We had calculated particle size using Scherer formula [12]. The particle sizes ( $3 \mathrm{~nm}$ and $5 \mathrm{~nm}$ ) obtained from the XRD patterns of $\mathrm{Ni} / \mathrm{PS} 10 \mathrm{~nm}$ and $20 \mathrm{~nm}$ films, respectively, were higher than those $(1 \mathrm{~nm}$ and $3 \mathrm{~nm})$ in plane Ni/PS films (10 $\mathrm{nm}$ and $20 \mathrm{~nm}$ thickness respectively). Also, the intensity of XRD peaks increases with increase in film thickness due to increase in crystallinity.

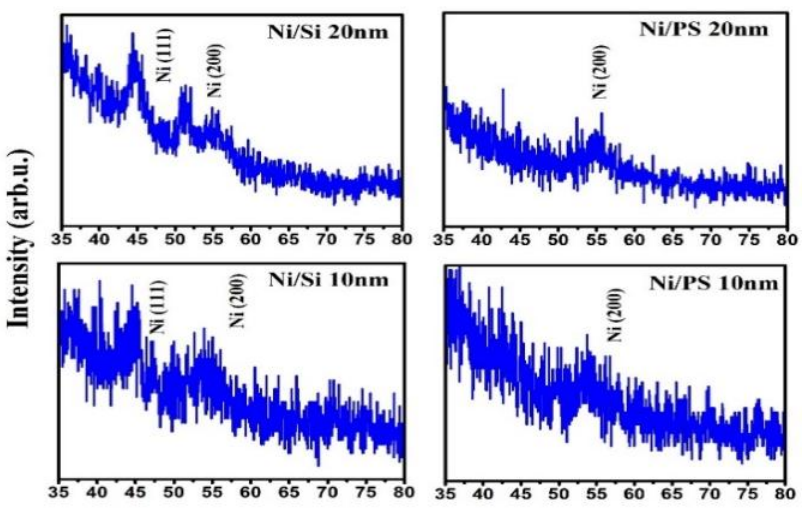

$2 \theta\left(^{\circ}\right)$

Fig. 3 - GI-XRD graphs of $\mathrm{Ni}(10 \mathrm{~nm}$ and $20 \mathrm{~nm})$ films deposited on PS and $\mathrm{Ni}(10 \mathrm{~nm}$ and $20 \mathrm{~nm})$ films deposited on $\mathrm{Si}$ substrates, respectively

\subsection{X-ray Reflectivity (XRR) Technique}

To understand the curvature induced modifications in the structural parameters like thickness and roughness of the deposited films, XRR measurements were done on all the samples and are presented in Fig. 4. The XRR data were fitted with Parratt formalism to extract different parameters from the experimental data. Both Ni/Si $(10 \mathrm{~nm}$ and $20 \mathrm{~nm})$ thin films are smooth and uniform as compare to Ni/PS thin films as indicated from the appearance of clear oscillations, so $\mathrm{Ni} / \mathrm{Si}$ XRR data could be well fitted. The fitted $\mathrm{Ni}$ thicknesses were 13 and $7 \mathrm{~nm}$ which are close to the designed/deposited thicknesses $(20 \mathrm{~nm}$ and $10 \mathrm{~nm})$ as 
shown in Table 1. Due to very high roughness, Ni/PS thin films data could not be fitted. We observed that when film thickness increases, roughness decreases very slightly in all the samples $(\sim 4 \AA$ to $\sim 3 \AA)$ which may be due to increase in crystallinity. Along with this, an increase in no. of Kiessig oscillations with film thickness was observed in the films deposited on $\mathrm{Si}$ substrate whereas an opposite behavior was observed in Ni/PS thin films, which may be attributed to the combination of the film roughness with the roughness of underlying substrate [7].

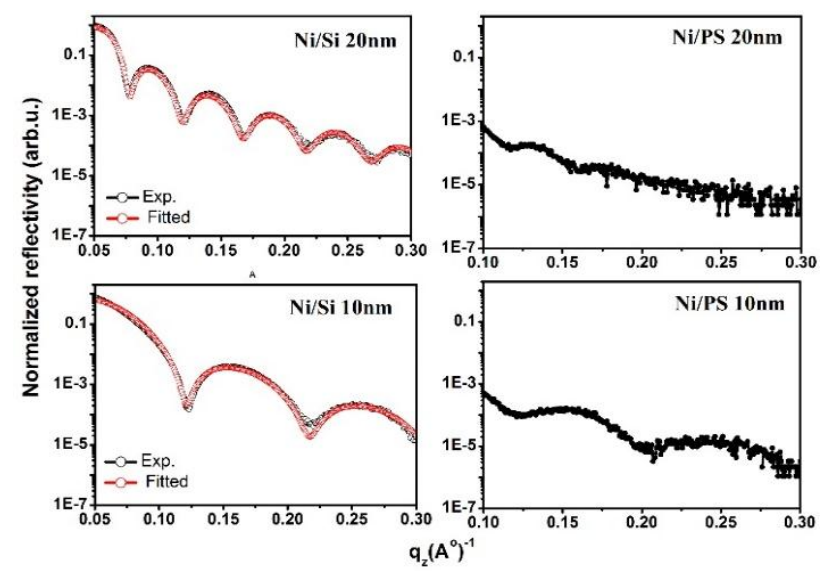

Fig. 4-XRR patterns of Ni thin films deposited on Si substrates and Ni thin films deposited on PS substrates

\subsection{Magnetic Measurements}

Fig. 5 shows the magnetic hysteresis loops of $\mathrm{Ni}$ thin films deposited on PS nanospheres and plain Si substrate. Corresponding magnetic parameters extracted from these loops are tabulated in Table 1. For these measurements, the magnetic field was kept in a direction parallel to the substrate. For films on plane substrate, the $\mathrm{Ni} / \mathrm{Si} 10 \mathrm{~nm}$ and $\mathrm{Ni} / \mathrm{Si} 20 \mathrm{~nm}$ films showed coercivity values as 2 Oe and 3 Oe, respectively. However, the coercivity increases drastically for the films on curved PS surface as compared to that on plane substrate (62 Oe and 89 Oe for the Ni/PS $10 \mathrm{~nm}$ and Ni/PS $20 \mathrm{~nm}$ thin film samples, respectively). In agreement with the structural observations, it may also be attributed to the surface roughness that affects the domain wall movement resulting in an enhancement in coercivity $[7,13]$.

The films on nanosphere arrays show very high coercivities with drastic changes in the loop shape compared to the films on plain substrate. This may be due to a modified reversal mechanism induced by the curved nature of underlying substrate and also due to the variations in microstructural parameters of the film like the change in size of grains and thickness distribution in the film plane [14]. Differences in the magnetic behaviors of these films were observed because of the variation in surface morphologies, structural parameters (grain size, roughness, shape and distribution of these grains), which play an important role in increasing the coercivity values and it can lead to a lot of pinning sites [15]. These pinning sites act as a barrier to the movement of the walls of the magnetic domain, thereby increasing the coercive field. Thus, it is clear that the coercive field changes as a result of the influence of combined effects including grain size, magnetic anisotropy and morphology of the surface that depends on the substrate and the thickness of the Ni film. The squareness ratio $\mathrm{S}$ (defined as the ratio of remnant magnetization and saturation magnetization) calculated from the hysteresis loops in terms of the ratio of the Kerr intensity at remanence to the Kerr intensity at saturation was also obtained. A small $S$ value may imply a rotation of magnetization while a large $S$ value is correlated with nucleation and motion of the domain wall. The squareness ratio of $\sim 1$ indicates that domain wall motion and nucleation are principal factors that take part in the magnetization reversal process [16-18]. For all the samples, squareness values are shown in Table 1. Ni/PS $10 \mathrm{~nm}$ and Ni/PS $20 \mathrm{~nm}$ squareness values were $\sim 1$ and 0.9 , respectively, and for samples $\mathrm{Ni} / \mathrm{Si} 10 \mathrm{~nm}$ and $\mathrm{Ni} / \mathrm{Si} 20 \mathrm{~nm}$, the squareness values were 0.89 and 0.88 , respectively.

A. Sharma et al. [16] and L. Kerkache [10] et.al found a value of $S$ equal to 0.82 and 0.91 for $\mathrm{Ni}$ $(40 \mathrm{~nm}) / \mathrm{Si}$; and $\mathrm{Ni}(42 \mathrm{~nm})$, which matches well with our results for $\mathrm{Ni} / \mathrm{Si}(10 \mathrm{~nm}$ and $20 \mathrm{~nm})$ films. These values are slightly different for curved films. It indicates that the variation of squareness does not depend on film thickness, however, it depends on the underlying substrate parameters.

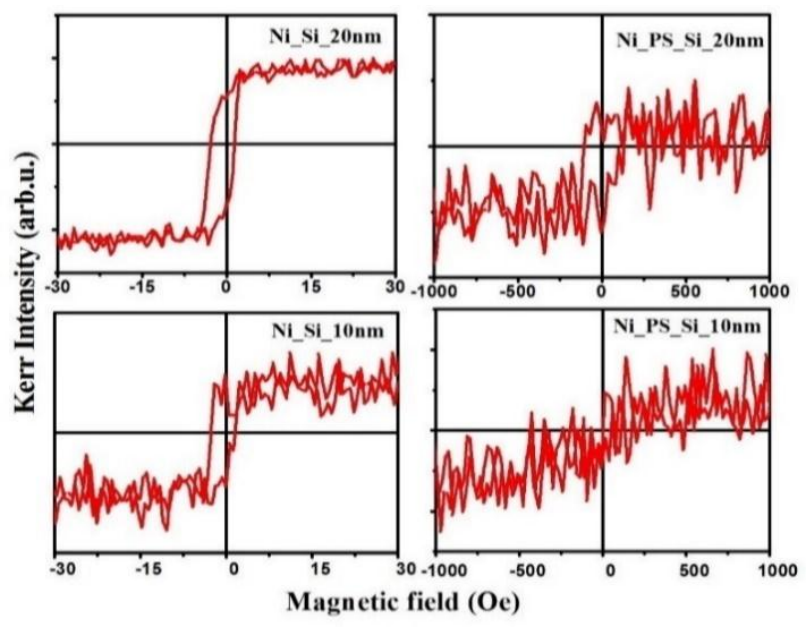

Fig. 5 - Recorded hysteresis loops of Ni (10 nm and $20 \mathrm{~nm})$ thin film deposited on PS and Si substrates

Table 1 - Structural and magnetic parameters extracted from various techniques

\begin{tabular}{|l|c|c|c|c|c|}
\hline Thin films & $\begin{array}{c}\text { Thick- } \\
\text { ness } \\
(d / \AA)\end{array}$ & $\begin{array}{c}\text { Rough- } \\
\text { ness } \\
(\sigma \AA)\end{array}$ & $\begin{array}{c}\text { Coer- } \\
\text { civity } \\
H_{c}(\mathrm{Oe})\end{array}$ & $\begin{array}{c}\text { Square- } \\
\text { ness } \\
M r / M s\end{array}$ & $\begin{array}{c}\text { Particle } \\
\text { size } \\
(\mathrm{nm})\end{array}$ \\
\hline $\mathrm{Ni} / \mathrm{Si} 20 \mathrm{~nm}$ & 130 & 3 & 3 & 0.88 & 3 \\
\hline $\mathrm{Ni} / \mathrm{PS} 20 \mathrm{~nm}$ & - & - & 89 & 0.90 & 5 \\
\hline $\mathrm{Ni} / \mathrm{Si} 10 \mathrm{~nm}$ & 70 & 4 & 2 & 0.89 & 1 \\
\hline $\mathrm{Ni} / \mathrm{PS} 10 \mathrm{~nm}$ & - & - & 62 & 1 & 3 \\
\hline
\end{tabular}

\section{CONCLUSIONS}

The effect of curvature and thicknesses on the structural and magnetic properties of Ni thin films was studied. For this, Ni thin film with the thicknesses of $10 \mathrm{~nm}$ and $20 \mathrm{~nm}$ were prepared by electron beam evaporation 
technique. The substrate surface for curved thin film deposition was prepared by fabricating self-assembled nanospheres array (800 $\mathrm{nm}$ diameter) coating onto plain Si (100). Reference films were simultaneously deposited on plain Si. Structural properties were investigated by using GIXRD, which showed enhancement in crystalline nature when the film thickness is increased from 10 to $20 \mathrm{~nm}$. XRR measurements showed modification in film oscillations and amplitude with film thickness. In addition, a higher coercivity was observed in the film deposited on PS substrate than that for the film on Si substrate.

\section{REFERENCES}

1. Williams Gwilym, Matthew Hunt, Benedikt Boehm, Andrew May, Michael Taverne, Daniel Ho, Sean Giblin, Nano Res. 11(2), 845 (2018).

2. J. Nogués, J. Sort, V. Langlais, V. Skumryev, S. Suriñach, J.S. Muñoz, M.D. Baró, Phys. Rep. 422, 65 (2005).

3. J.P. Wang, Nat. Mater. 4, 191 (2005).

4. Y.J. Chen, J.P. Wang, E.W. Soo, L. Wu, T.C. Chong, J. Appl. Phys. 91, 7323 (2002).

5. A. Chalastaras, L.M. Malkinski, J.-S. Jung, S.-L. Oh, J.-K. Lee, C.A. Ventrice, V. Golub, G. Taylor, IEEE Trans. Magn. 40 No 4, 2257 (2004).

6. J.C. Hulteen, R.P. Van Duyne, J. Vac. Sci. Technol. A 13, 1553 (1995).

7. Y. Kumar, J. Tripathi, D. Kumar, P. Gupta, S. Tripathi, A. Sharma, AIP Conf. Proc. 2265, 030303 (2020).

8. M. Hemmous, A. Layadi, L. Kerkache, N. Tiercelin, V. Preobrazhensky, P. Pernod, Metallur. Mater. Trans. A 46 No 9, 4143 (2015).

9. J.H. Yang, N.N. Yang, Y.X. Wang, Y.J. Zhang, Y.M. Zhang, Y. Liu, M.B. Wei, Y.T. Yang, R. Wang, S.Y. Yang, Solid State Commun. 151, 1428 (2011).

\section{ACKNOWLEDGEMENTS}

The authors are thankful to UGC-DAE-CSR, Indore (M.P.), India (Ref. No. CSR-IC/CRS-148/2015-16/05 dated 26/03/2016) for providing financial grant for research work. Authors are also thankful to Dr. Dileep Kumar for electron beam deposition, Dr. Pooja Gupta for MOKE and Mr. Anil Gome for GIXRD and XRR measurements. We are also grateful to sophisticated analytical instrument facility (SAIF), Manipal University Jaipur for FESEM measurement.

10. L. Kerkache, A. Layadi, M. Hemmous, A. Guittoum, M. Mebarki, N. Tiercelin, A. Klimov, V. Preobrazhensky, P. Pernod, SPIN 9 No 01, 1950006 (2019).

11. L.A. García-Cerda, K.M. Bernal-Ramos, Sagrario M. Montemayor, M.A. Quevedo-López, R. Betancourt-Galindo, D. Bueno-Báques, J. Nanomater. 2011, 162495 (2011).

12. A. Sharma, J. Tripathi, Yogesh Kumar, G.S. Chandrawat, R. Bisen, S. Tripathi, J. Nano- Electron. Phys. 12 No 2, 02040 (2020).

13. Y-P. Zhao, R.M. Gamache, G-C. Wang, T-M. Lu, G. Palasantzas, J.Th. M. De Hosson, J. Appl. Phys. 89 No 2, 1325 (2001).

14. A. Sharma, J. Tripathi, S. Tripathi, Yogesh Kumar, K.C. Ugochukwu, D. Kumar, M. Gupta, R.J. Chaudhary, J. Magn. Magn. Mater. 510, 166599 (2020).

15. J. Pflaum, E. Hubner, Th. Zeidler, T. Schmitte, J. Pelzl, Thin Solid Films 318, 186 (1998).

16. A. Sharma, S. Tripathi, K.C. Ugochukwu, J. Tripathi, Thin Solid Films 536, 249 (2013).

17. J. Swerts, K. Temst, N. Vandamme, C. Van Haesendonck, Y. Bruynseraede, J. Magn. Magn. Mater. 240, 380 (2002).

18. L. Nzoghé-Mendome, A. Aloufy, J. Ebothé, Mol. Cryst. Liq. Cryst. 555, 32 (2012). 